\section{EYE-STRAIN IN RELATION TO THE GENERAL HEALTH.}

By JAMES HINSHELWOOD, M.A., M.D. GLASG., SURGEON TO GLASGOW EYE INFIRMARY, AND LECTURER ON OPHTHALMOLOGY IN THE GLASGOW WESTERN MEDICAL SCHOOL.

EVERYTHING appertaining to the well-being of the patients rander his care is of importance to the general practitioner. With regard to eye symptoms, however, he is too apt to regard these as of importance only to the specialist and therefore outside of his sphere of interest. I wish, therefore, to direct attention to the close relationship which frequently exists between eye-strain and the general health, how these influence one another, and how therefore the practitioner must be familiar with the far-reaching effects of eye-strain if he would deal successfully with these sufferers, who will generally apply to him in the first place for the rrelief of their distressing symptoms.

Eye-strain frequently acts by exhausting the nervous system of the patient, and hence through the medium of ibrain and spinal cord often has a profound and far-reaching iffect on the functions of the various organs of the body and wn the general nutrition of the patient.

am not one of those who would attribute "all the ills that flesh is heir to " directly to the varied effects of eye-strain. There has been a tendency on the part of some writers, especially in America, to exaggerate the far-reaching effects of eye-strain, and this has given rise to a certain amount of discredit and scepticism regarding the subject. But as experience becomes wider one becomes more and more impressed with the fact that eye-strain has often a most prejudicial effect on the general health of the patient, and that great improvement in this may often be brought about simply by the relief of the eye-strain.

An important thing to bear in mind is that whilst eyestrain often manifests itself by pain or discomfort in the eye itself, this is not always the case. In many cases the eyestrain is accompanied by no pain or discomfort in the eye, but by symptoms altogether outside of the eye itself; and these are the cases which often escape the notice of the practitioner unless his attention has been specially directed to the subject. It will be well for him, therefore, to thoroughly familiarise himself with these symptoms of eyestrain, which arise apart from the eye itself, and this knowledge will often be of the greatest service in ministering to the well-being of the patients under his charge.

Another most important thing for the practitioner to bear in mind is that the symptoms which $I$ am about to describe as arising from eye-strain may be due to a very small error of refraction, and hence may never be suspected either by the physician or by the patient. I think this is peculiarly true of astigmatism, where a very small error, even $0.25 \mathrm{D}$., may give rise to distressing symptoms. In fact, my experience is that the small amounts of astigmatism give rise to symptoms of discomfort more frequently than the high degrees. The explanation, I think, is that a person with a high degree of astigmatism makes no effort to see better, as his vision is so defective, but that the smaller degrees of astigmatism, only causing a slight blurring, induce the patient to make a continuous accommodative effort to counteract it and get clear images, and hence cause continuous ciliary strain. These patients with slight degrees of astigmatism have good vision, and many of them pride shemselves on their good vision, and are rather indignant at the idea of their symptoms being due to eye-strain. Patients with hypermetropia and suffering severely from eyestrain may have absolutely normal vision if tested by the test fypes, and it is this fact which completely misleads frequently both the patient and physician as to the true acause of their symptoms.

Another reason why eye-strain, as a cause of symptoms, is often overlooked is because the patient is already wearing glasses and has derived little or no benefit from them. It cannot be too strongly emphasised that to derive benefit the refractive error must be exactly and properly corrected. In most cases this cannot be satisfactorily accomplished without examination of the patient under homatropine or atropine. Whis can be properly done only by an ophthalmic surgeon, and nothing requires greater care than the fitting of patients with appropriate glasses. In many cases, as when there is marked inequality of refraction in the two eyes, the problem is a very difficult one and requires great care, thought, and experience.

Of all the reflex symptoms of eye-strain, the most frequent is headache, and yet very many cases of ocular headache go on suffering for years without the true cause being suspected. It is important to bear in mind the points with relation to eye-strain which I have already mentioned-viz., that eyestrain may be present without the patient experiencing any discomfort or pain in the eyes; that eye-strain may be present although the patient possesses good and even normal vision as tested by the types; and that glasses will not give relief unless the refractive error has been adequately and exactly corrected. Bearing in mind these facts, it is evident that the only safe rule of practice is that every patient suffering from headache of a recurring or chronic character should have his refraction carefully examined by someone thoroughly competent to make such an examination.

The ocular headache may occupy any position-frontal, vertical, or occipital, and I have frequently seen pain even at the back of the neck due to eye-strain. Whilst the situation of the headache does not guide us to the diagnosis, in the majority of cases it is frontal or vertical. Ocular headache is generally absent in the morning. If the patient has had a good night's sleep he wakens up free from headache. It begins in the course of the day and gradually increases in intensity as the day goes on. This is always the case with a pure ocular headache. This is in contrast to the character of the toxic headache, which is worst in the morning after a good night's sleep, and becomes better when the patient gets up and exerts himself. The toxic elements accumulate in the blood during sleep and exercise helps in their elimination. In many cases of headache we have a combination of the toxic and ocular, and in such cases the patient wakens with a headache, which, however, gets worse as the day goes on. In such cases the cure of the patient can only be accomplished by the relief of the eye-strain and the removal of the toxæmia, of which constipation is a frequent cause. Anæmia and eye-strain are also a frequent combination in the production of headache, and cure can only be brought about by the removal of eye-strain and the internal administration of drugs for the cure of the anæmia.

This complex origin of headaches is a point that is very frequently lost sight of, attention being given to one factor only in the ease. It is to be remembered that whilst eyestrain is frequently the sole cause of headache, there are other cases where the eye-strain is only one factor in its production. The relief of the eye-strain will benefit the patient, but complete relief will not be given until all the causal factors have been dealt with and removed.

It is very remarkable how much the general health of the patient benefits by the removal of a chronic headache. Even if the headache has not been very intense, its frequent and often daily presence produces in the patient great mental depression, which through the influence of the nervous system has a powerful influence on the nutrition of the body as a whole. The complete removal of this headache, which has made the patient's life miserable, lifts him into a new world, and the general condition of the patient, both mental and physical, is enormously benefited thereby.

In addition to definite headache patients often complain of peculiar sensations in the head, which disappear on the relief of eye-strain and which are undoubtedly due to it. $\mathbf{A}$ feeling of pressure or tension in the head is the most common of the head sensations apart from pain. Less frequently I have heard patients complain of a feeling of tingling or crawling over certain areas of the head, and in some cases the patients could not venture to describe the sensation further than remarking that there was a very peculiar feeling in the head, always produced by the use of the eyes.

$I$ have seen several cases of distressing insomnia which were directly due to eye-strain and which disappeared completely when the eye-strain was removed. The most striking case of this kind I saw about a year ago. The patient suffered so much that he became neurasthenic with lowering of the general nutrition of the body, and had been away from his work entirely for about 
four months during the preceding winter. He was a hypermetrope with astigmatism and was engaged at an occupation where there was great and continuous eye-strain. At night after his day's work he was so exhausted that he was unable to do anything, and when he went to bed he could not sleep. This state of affairs gradually impaired his general nutrition and especially exhausted his nervous system, so that he felt himself unable for his work and was away from it for four months. There being both hypermetropia and astigmatism, there was a constant strain on his accommodation, so that this patient suffered severely. I prescribed two pairs of glasses for him, one for constant wear and the other for near work, which put his accommodation in a state of rest. It was very remarkable to see the speedy improvement in this patient's condition. He had no longer the sense of exhaustion after his day's work and got a good night's sleep after going to bed. This brought about a very rapid improvement in the patient's general well-being and condition. Since wearing his glasses he has been able to go on steadily with his work without any difficulty.

I have seen several cases where the patients had been using hypnotics for considerable periods, but when their refractive error was properly corrected and eye-strain completely relieved they were able to dispense entirely with their hypnotics in a very short period of time. In all patients using the eyes much for near work and suffering from insomnia eye-strain should always be thought of as a possible cause.

The condition of nervous exhaustion commonly known amongst brain workers as "brain-fag" is very frequently simply due to eye-strain. Those engaged in pursuits which necessitate the close and continuous use of the eyes for reading and writing often experience a sense of prostration and exhaustion, frequently described as brain-fag, nerve exhaustion, and neurasthenia. The period during which the individual can work may become gradually more limited until he is wholly incapacitated from further effort by this increasing sense of exhaustion and prostration. In such cases I would strongly urge the very careful examination of the refraction of the eye. In such patients reading and writing constantly from eight to twelve hours a day, a very slight refractive error often produces a peculiar sense of exhaustion which is mistaken for cerebral exhaustion. I speak from experience of the great benefit derived in many such eases by the correction of refractive errors, often very slight. The relief of the eye-strain soon led to the disappearance of the sense of exhaustion at the end of the day's work and what had been for years regarded as "brain-fag" was seen to have been in reality " eye-fag."

Choreiform movements of the facial muscles in children are frequently due to eye-strain and disappear when the eyestrain is relieved by the prescription of a suitable glass. The spasmodic movements are most frequently met with in, and sometimes confined to, the orbicularis palpebrarum muscles, so that the patient is constantly blinking, and is often thought to be suffering from a mild attack of chorea. A large proportion of such cases are due to eye-strain, and when this is so the spasmodic movements will be greatly relieved or even abolished temporarily by the use of atropine, but permanent relief is only obtained by the wearing of suitable glasses. Although the spasmodic movements are often confined to the orbicularis muscles, they may extend to the facial muscles, and these cases are even more likely to be mistaken for cases of chorea.

Vertigo is another symptom sometimes produced by eyestrain. Most are familiar with vertigo as a symptom of cerebral disease, of aural disease, of gastric disturbance, and of several diseases of the nervous system. We are all familiar also with ocular vertigo, due to paralysis or paresis of an ocular muscle, and which always disappears on the closure of one eye. Few, however, think of the possibility of vertigo as the result of eye-strain. I have seen two cases where this was so severe that the patients were supposed to be suffering from disease of the nervous system. In all the cases of vertigo I have seen due to eye-strain it has been associated with hypermetropia or hypermetropic astigmatism, and rapidly disappeared after relief of the eye-strain by the prescription of appropriate glasses. In all cases of vertigo, therefore, which cannot be traced to a definite cause, and which do not yield to treatment, eye-strain ought to be thought of as a possible cause, and the patient's refraction carefully examined and any defect corrected. Eye-strain sometimes plays an important part as a causal factor in dyspepsia, and many cases of dyspepsia, though relieved by drugs and dieting, will not be cured until the factor of eye-strain is completely eliminated by the prescription of suitable glasses for the patient. The nervous system plays a most important part in the causation of many forms of dyspepsia, and it is frequently through the exhaustion of the nervous system by prolonged eye-strain that the functions of the stomach are deranged. It is in the acid form of dyspepsia especially that I have found eye-strain play an important part. In this form of dyspepsia the discomfort comes on a considerable time after food and is relieved by food. This form of dyspepsia is nearly always associated with a lowered tone of the nervous system. It is an exceedingly obstinate form of dyspepsia to treat, as drugs seem to have little effect on it apart from the temporary relief of symptoms. Cure is to be brought about only by an improved tone of the nervous system, and in some cases the relief of the nervous exhaustion produced by eyestrain is the only possible way of effecting this and so curing the patient.

I have said nothing of the local manifestations of eyestrain in the eye or its appendages. These are well known, and their very presence at once directs the attention both of patient and physician to eye-strain as their probable cause. I have in these observations limited myself to discussing the symptoms of eye-strain outside the local manifestations, with which I have become familiar in my daily work as an ophthalmic surgeon. My purpose has been to call attention to conditions of ten profoundly affecting the general health of the patients which are not sufficiently known to the practitioner as possible results of eye-strain, and yet where the removal of this factor is in many cases the only possible means of giving the patients relief. Glasgow.

\section{DIFFUSE LATENT LABYRINTHITIS : ITS DANGERS IN THE RADICAL MASTOID OPERATION.}

By JAMES haRPhR, M.A., M.B., CH.B. GlasG., FORMERLY HOUSE SURGEON TO THE HOSPITAL FOR DISEASES OF THE THROAT, GOLDEN-SQUARE, LONDON.

AMONG major surgical operations that known as the radical mastoid operation is considered to be one of those which may be performed with practically no danger to the life of the patients. Large numbers of this operation are performed yearly with such gratifying results that operators are inclined to be lulled into a false sense of security and to point to what are unfortunately in some cases only apparent successes as proof of the almost non-existence of danger.

All surgeons of experience, whether their work brings them into contact with aural work or not, recognise the grave danger there lies in aural suppuration as regards intracranial complications. They know the intimate connexion there is between these two conditions, and when in the course of aural suppuration symptoms arise which point to a suspicion of intracranial mischief they do not hesitate in advising more radical treatment for the ear than that which it has been receiving.

Another type of case for which advice is continually being sought is that of a patient who for a number of years has had a discharging ear. All the recognised methods of conservative treatment suitable to the case have been tried without success, and the patient now seeks advice as to whether something cannot be done to rid him once and for all of this troublesome affliction. If the hearing be good the surgeon may counsel waiting a little longer, but if, as is the general rule, the hearing has gone or has practically disappeared, the treatment generally advised is the radical mastoid operation. Having tendered this advice the surgeon is asked the usual questions: Is there any danger and will this operation stop the discharge?

The answer to the first question is that with the exception of the risk necessarily attached to an anæsthetic the danger to life is practically non-existent. The answer to the second question is in the affirmative. These are the two replies usually given. Yet most of us who have had experience of aural work, or who have been following the literature of the 